Case Report

\title{
Treatment of T-Cell Prolymphocytic Leukemia with Central Nervous System Involvement Using Intrathecal Alemtuzumab Administration
}

\author{
Jinichi Mori $\left(\mathbb{D},{ }^{1}\right.$ Kumi Oshima, ${ }^{1,2}$ Satoshi Kimura, ${ }^{3}$ and Takayuki Ikezoe ${ }^{3}$ \\ ${ }^{1}$ Department of Hematology, Jyoban Hospital, Tokiwa Foundation, Fukushima, Japan \\ ${ }^{2}$ Patient Safety Division, QI Center, St. Luke's International Hospital, Tokyo, Japan \\ ${ }^{3}$ Department of Hematology, Fukushima Medical University, Fukushima, Japan
}

Correspondence should be addressed to Jinichi Mori; jinichimori@gmail.com

Received 5 June 2020; Revised 13 July 2020; Accepted 15 July 2020; Published 27 July 2020

Academic Editor: Vincent Ribrag

Copyright (C) 2020 Jinichi Mori et al. This is an open access article distributed under the Creative Commons Attribution License, which permits unrestricted use, distribution, and reproduction in any medium, provided the original work is properly cited.

T-cell prolymphocytic leukemia (T-PLL) is a rare hematologic cancer with a dismal prognosis. Although a small number of patients have central nervous system (CNS) involvement, a standard treatment approach for these patients has not been established. Herein, we present a case of T-PLL with CNS involvement that was treated with a higher dose of intrathecal alemtuzumab than that previously reported. A 66-year-old man who had T-PLL with CNS involvement was admitted to our hospital. Intravenously administered alemtuzumab, a monoclonal antibody against the CD52 antigen, successfully reduced leukemia cells in peripheral blood; however, intrathecal treatment with methotrexate, cytarabine, and prednisone had a limited effect on the CNS involvement. Therefore, we intrathecally injected alemtuzumab as an experimental treatment. Although we escalated the dose of intrathecal alemtuzumab up to $5 \mathrm{mg}$, no adverse reaction was noted; however, this treatment did not completely clear the leukemia cells from the patient's cerebrospinal fluid (CSF). We performed whole brain and whole spinal irradiation therapies and subsequently performed allogeneic transplantation from a human leukocyte antigen-matched sibling donor with a conditioning regimen containing total body irradiation. At 21 days after transplantation, leukemia cells remained in his CSF. Although intrathecal alemtuzumab did not eliminate the CNS-invading leukemia cells, it was well-tolerated in our case. Further accumulation of similar cases is needed to determine the benefits and safety of intrathecal alemtuzumab administration.

\section{Introduction}

T-cell prolymphocytic leukemia (T-PLL), a rare hematologic cancer, is associated with poor patient prognosis; the overall 3-year survival rate is $40 \%$ after allogeneic hematopoietic stem-cell transplantation (HSCT) in patients who have previously received conventional chemotherapies [1]. Induction therapy with alemtuzumab, a humanized monoclonal antibody against the CD52 antigen, and subsequent allogeneic HSCT may promote longer survival; however, overall, the 4 -year survival rate remains at $56 \%$ [2]. Few reports have described central nervous system (CNS) involvement, a dismal prognostic factor, in patients with T-PLL [3-5]. For transplantation candidates, pre-HSCT control of CNS involvement is needed to achieve improved transplantation outcomes, as in other types of leukemia [6]; however, a standard treatment approach for cases of T-PLL with CNS involvement does not exist. To date, only one previous report has demonstrated a case where $3 \mathrm{mg}$ of intrathecal alemtuzumab was safely administered; however, the efficacy of this treatment was not evaluated in that case. Here, we present a case of T-PLL with CNS involvement that was treated with up to $5 \mathrm{mg}$ of intrathecal alemtuzumab, but was not eliminated.

\section{Case Presentation}

A 66-year-old man was diagnosed with T-PLL in 2011. At the time of diagnosis, he was asymptomatic and had a 
peripheral white blood cell (WBC) count of $17,600 \mu \mathrm{L}$, including an abnormal lymphocyte count of $15,000 \mu \mathrm{L}$. Thus, his attending physician chose an observant waiting approach. The patient's lymphocyte count gradually increased and reached $30,000-40,000 \mu \mathrm{L}$ in 2018 . He developed night sweats, fatigue, low-grade fever, and headaches. Computed tomography revealed hepatosplenomegaly, and in 2019, he was moved to our hospital to initiate treatment. At the time of the transfer, his WBC count was $52,600 \mu \mathrm{L}$, including a lymphocyte count of $46,800 \mu \mathrm{L}$. Microscopic examination of the blood smear revealed small-to-medium-sized lymphocytes that featured irregularly shaped nuclei and condensed chromatin (Figure 1).

The lymphocytes exhibited a post-thymic T-cell phenotype ( $\mathrm{Tdt}^{-}, \mathrm{CD}^{-}, \mathrm{CD}^{+}, \mathrm{CD}^{+}$, and $\left.\mathrm{CD}^{+}\right)$and harbored chromosomal abnormalities (add(15)(q24) and $\operatorname{del}(7)(\mathrm{p} ?))$. As the patient was experiencing headaches, we performed a cerebrospinal fluid (CSF) examination and found leukemic lymphocytes carrying the same pattern of surface antigens as those in the peripheral blood. As the initial chemotherapy (fludarabine and cyclophosphamide) caused tumor lysis syndrome, we discontinued the treatment and started administering intravenous alemtuzumab after the resolution of the tumor lysis syndrome. Following these changes, the patient's lymphocyte count dropped sharply (Figure 2).

We concurrently treated the CNS involvement with a dose of intrathecal methotrexate and followed it by intrathecal therapy containing methotrexate, cytarabine, and prednisone weekly. However, the number of CNSinvading leukemic cells reached the lowest point after the fourth intrathecal injection, and the number of leukemic cells in the patient's CSF increased. Once we received approval from our institutional review board, we initiated an experimental treatment with intrathecal alemtuzumab injections. The patient did not experience any adverse reactions to the intrathecal alemtuzumab injections. To eliminate the leukemia cells in the patients' CSF, we increased the intrathecal alemtuzumab dose to $5 \mathrm{mg}$; however, the cells did not completely disappear. We performed whole brain and whole spinal irradiation therapies and subsequently performed allogeneic transplantation from an HLA-matched sibling donor with a conditioning regimen containing total body irradiation. At 21 days after transplantation, 23 leukemia cells $\mu \mathrm{L}$ remained in his CSF. The patient is currently receiving best supportive care.

\section{Discussion}

We demonstrated here a patient with T-PLL and CNS involvement that was treated with intrathecal alemtuzumab. This treatment unfortunately did not clear the CNS-invading leukemia cells but allowed us to bridge to allogeneic transplantation without any adverse reactions. To the best of our knowledge, this is the first report of a case in which the efficacy and tolerance to intrathecal

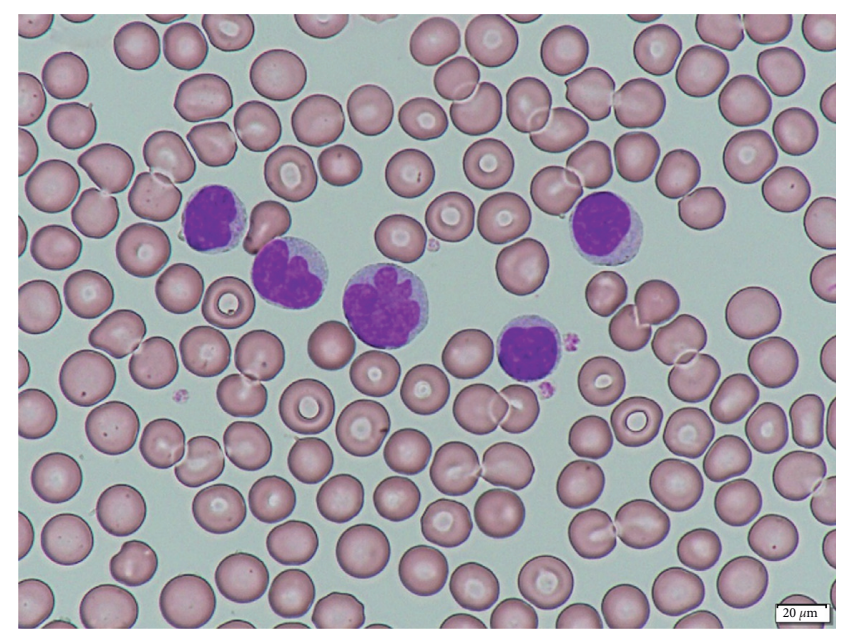

Figure 1: Microscopic image of leukemic lymphocytes in the peripheral blood smear.

alemtuzumab injections were evaluated. In a previous report by Alsawah et al., the authors claimed successful treatment with $3 \mathrm{mg}$ of intrathecal alemtuzumab for CNS involvement that was refractory to prior treatment with intrathecal cytarabine and methotrexate and whole brain irradiation [7]. However, when intrathecal alemtuzumab administration was initiated in their case, the patient's CSF leukemic cell count was $1 \mu \mathrm{L}$, limiting the evaluation of the treatment efficacy. In contrast, in our case, the patient had a CSF leukemic cell count of $3 \mu \mathrm{L}$ at the initiation of intrathecal alemtuzumab treatment. Unfortunately, our patient's CNS involvement did not resolve, despite the use of a higher dose $(5 \mathrm{mg})$ of intrathecal alemtuzumab than that previously reported.

Several in vitro studies have demonstrated that alemtuzumab exerts its antitumor effect by both complement-dependent cytotoxicity (CDC) and antibodydependent cellular cytotoxicity (ADCC) [8-10]. Although the effect in vivo has not been studied extensively, a preclinical study using human CD52 transgenic mice showed that ADCC plays a crucial role in the elimination of CD52-positive lymphocytes [11]. The study also demonstrated that intravenous administration of alemtuzumab cleared CD52-positive lymphocytes from the peripheral blood but not from certain organs (spleen and thymus) that contain fewer ADCC effector cells, such as neutrophils, natural killer cells, and macrophages. In our patient, intrathecally injected alemtuzumab might have provoked weak CDC and no ADCC in the CSF, which had complement levels that were 100-200-fold lower than those in the serum and extremely few ADCC effector cells. Thus, we speculate that this resulted in the lack of a significant response to the CNS-invading T-PLL cells [12].

Although the efficacy of intrathecal alemtuzumab therapy was limited in our case, it was well-tolerated even at a dose of $5 \mathrm{mg}$. Further information from similar cases is needed to determine the benefits and safety of this treatment. 


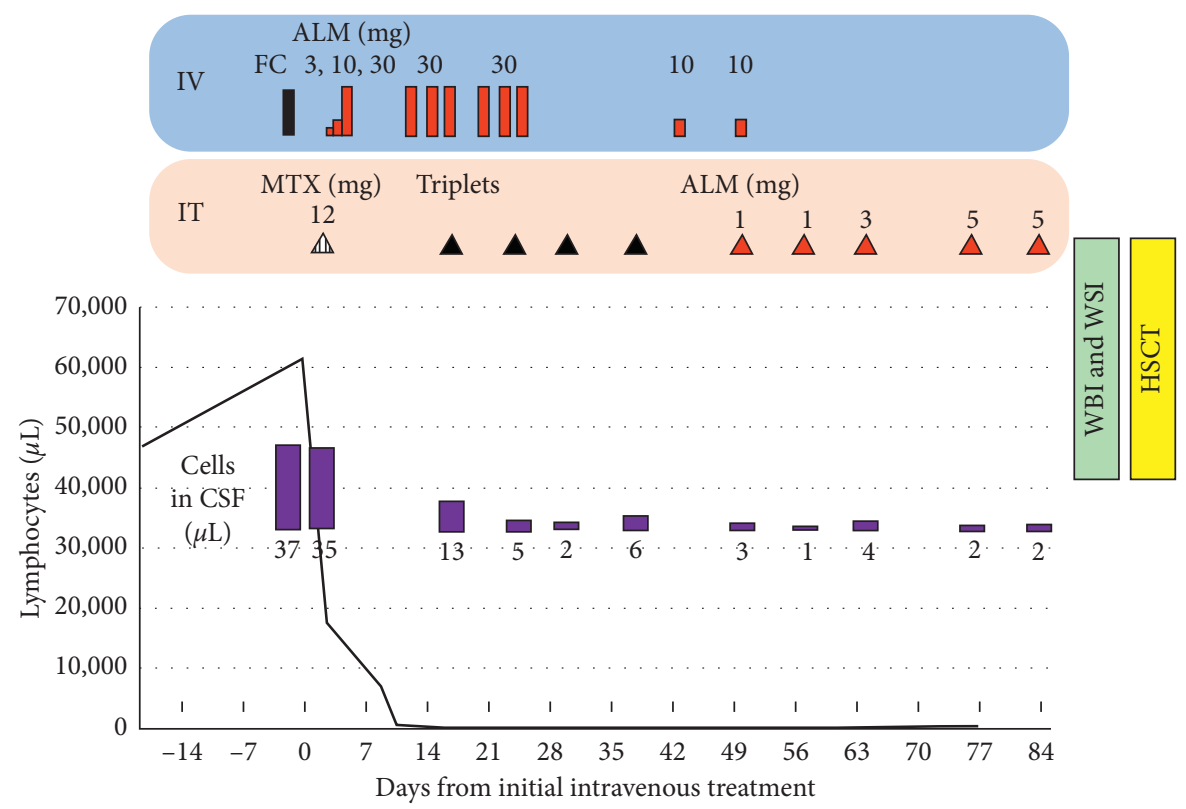

FIgURE 2: Treatment time course for the leukemia and central nervous system involvement. ALM: alemtuzumab; CSF: cerebrospinal fluid; FC: fludarabine and cyclophosphamide; HSCT: hematopoietic stem-cell transplantation; IT: intrathecal treatment; IV: intravenous treatment; MTX: methotrexate; WBI: whole brain irradiation; and WSI: whole spine irradiation. Triplets consist of $15 \mathrm{mg}$ of methotrexate, $40 \mathrm{mg}$ of cytarabine, and $20 \mathrm{mg}$ of prednisolone.

\section{Data Availability}

In accordance with the provisions of the ethics committee, personal information about the patient cannot be disclosed.

\section{Ethical Approval}

The treatment protocol in this study was approved by our institutional review board and conducted in accordance with the Declaration of Helsinki.

\section{Consent}

We obtained written informed consent for the experimental procedure and publication of the research results.

\section{Conflicts of Interest}

The authors have no conflicts of interest to declare.

\section{References}

[1] S. Yamasaki, H. Nitta, E. Kondo et al., "Effect of allogeneic hematopoietic cell transplantation for patients with T-prolymphocytic leukemia: a retrospective study from the Adult Lymphoma Working group of the Japan society for hematopoietic cell transplantation," Annals of Hematology, vol. 98, no. 9, pp. 2213-2220, 2019.

[2] B. R. Dholaria, E. Ayala, L. Sokol et al., "Allogeneic hematopoietic cell transplantation in T-cell prolymphocytic leukemia: a single-center experience," Leukemia Research, vol. 67, pp. 1-5, 2018.

[3] F. Brito-Babapulle, D. Huang, P. Lavender, D. Galton, and D. Catovsky, "Regression of intracerebral lesions in T prolymphocytic leukaemia treated with intravenous deoxycoformycin," European Journal of Haematology, vol. 40, no. 2 , pp. $185-187,1988$.

[4] L. Garderet, H. Bittencourt, A. Kaliski et al., "Treatment of T-prolymphocytic leukemia with nonmyeloablative allogeneic stem cell transplantation," European Journal of Haematology, vol. 66, no. 2, pp. 137-139, 2001.

[5] U. Y. Malkan, G. Gunes, O. Yayar, H. Demiroglu, A. Yesilirmak, and A. Uner, "A T-cell prolymphocytic leukemia case with central nervous system involvement," International Journal of Clinical and Experimental Medicine, vol. 8, no. 8, pp. 14207-14209, 2015.

[6] S. Ikegawa, N. Doki, S. Kaito et al., "Central nervous system involvement at the time of allogeneic hematopoietic stem cell transplantation is associated with a poor outcome in patients with acute myeloid leukemia," Pathology \& Oncology Research, vol. 23, no. 2, pp. 433-437, 2017 Apr.

[7] F. Alsawah, L. Benitez, S. Choi et al., "Intrathecal alemtuzumab: a potential treatment of refractory leptomeningeal T-cell prolymphocytic leukemia," Blood Advances, vol. 3, no. 21, pp. 3333-3336, 2019.

[8] C. S. Zent, C. R. Secreto, B. R. LaPlant et al., "Direct and complement dependent cytotoxicity in CLL cells from patients with high-risk early-intermediate stage chronic lymphocytic leukemia (CLL) treated with alemtuzumab and rituximab," Leukemia Research, vol. 32, no. 12, pp. 1849-1856, 2008.

[9] J. Golay, M. Manganini, A. Rambaldi, and M. Introna, "Effect of alemtuzumab on neoplastic B cells," Haematologica, vol. 89, no. 12, pp. 1476-1483, 2004.

[10] R. I. Cruz, F. J. Hernandez-Ilizaliturri, S. Olejniczak et al., "CD52 over-expression affects rituximab-associated complement-mediated cytotoxicity but not antibody-dependent cellular cytotoxicity: preclinical evidence that targeting CD52 with alemtuzumab may reverse acquired resistance to rituximab in non-Hodgkin lymphoma," Leukemia \& Lymphoma, vol. 48, no. 12, pp. 2424-2436, 2007. 
[11] Y. Hu, M. J. Turner, J. Shields et al., "Investigation of the mechanism of action of alemtuzumab in a human CD52 transgenic mouse model," Immunology, vol. 128, no. 2, pp. 260-270, 2009.

[12] M. Trbojević-Cepe, V. Brinar, M. Pauro, Z. Vogrinc, and N. Stambuk, "Cerebrospinal fluid complement activation in neurological diseases," Journal of the Neurological Sciences, vol. 154, no. 2, pp. 173-181, 1998. 